\title{
Relación entre la edad del profesorado de música andaluz y el desarrollo de la Escuela TIC 2.0
}

\section{The relationship between the age of music teachers in Andalucía and the development of the IT program Escuela TIC 2.0}

\author{
Emilia MARTOS SÁNCHEZ1 ${ }^{1}$ Purificación PÉREZ-GARCÍA² y \\ Julia BERNAL VÁZQUEZ ${ }^{2}$ \\ ${ }^{1}$ IES Rosa Navarro de Olula del Río de Almería (España) y \\ ${ }^{2}$ Universidad de Granada
}

Recibido: Febrero 2015

Evaluado: Marzo 2015

Aceptado: Junio 2015

\begin{abstract}
Resumen
El objetivo de este artículo era conocer si existía relación entre la edad del profesorado andaluz de música de Educación Secundaria Obligatoria (ESO) y el desarrollo del programa andaluz Escuela TIC 2.0. Para esta investigación, la muestra estuvo compuesta por 207 docentes de música de la ESO, quienes cumplimentaron un cuestionario sobre los diferentes aspectos que conllevaba dicho programa en conexión con su área, tales como la formación en TIC, las tecnologías en el aula, la actitud hacia las TIC y las competencias, los recursos digitales o la vinculación entre las familias y la Escuela TIC. Dicho cuestionario fue sometido tanto a la validación estadística por componentes principales como a la validación de jueces y a procedimientos de fiabilidad mediante el alpha de Cronbach y la metodología de las dos mitades. Se aplicó un análisis de contingencias y los resultados mostraron que cuanto menor era la edad, mayor era el uso y valoración de las TIC y viceversa. El estudio reveló que, efectivamente, la edad del profesorado de música andaluz de la ESO estaba relacionada con la determinación de incorporar las TIC y el desarrollo del programa Escuela TIC 2.0.
\end{abstract}

Palabras clave: TIC, edad, enseñanza secundaria, música.

\begin{abstract}
The object of this paper was to discover if there was a relationship between the age of music teachers in compulsory secondary education (ESO) and the development of the Andalusian ICT school program Escuela TIC 2.0 [ICT School 2.0]. For this investigation a sample composed of 207 secondary education music teachers participated fulfilling a questionnaire on the different aspects of the program in connection to their area, such as their training in ICT, the technology available in the classrooms, the attitude towards ICT and competencies, digital resources and the ties between the families and the ICT School. This questionnaire was subjected both to a
\end{abstract}


statistical validation of its principal components and at the same time to a validation by judges, and to reliability procedures using Cronbach's Alpha and the Split-half methodology. A contingency analysis was applied and the results showed that the lower the age, the higher was the use and the evaluation of ICT, and vice versa. This study demonstrated that the age of music teachers in secondary schools in Andalusia is directly related to the determination to incorporate ICT and to the development of the Escuela TIC 2.0 program.

Keywords: ICT, age, secondary education, music.

Este artículo tiene como finalidad determinar si el factor de la edad del profesorado, de música, de Educación Secundaria Obligatoria (ESO) de Andalucía, está relacionado con el desarrollo del último programa educativo sobre las tecnologías de la información y la comunicación, puesto en funcionamiento por el gobierno de la comunidad autónoma de Andalucía. Nos referimos a la Escuela TIC 2.0.

\section{Introducción y estado de la cuestión}

La web 2.0 supone una auténtica revolución que afecta a muchos sectores. La expresión 2.0 atribuida a movimientos del mercado global, tal es el caso de Empresa 2.0, Educación 2.0 o E-learning 2.0, se utiliza como signo de modernidad e innovación que, en opinión de Castaño, Palacio, Maíz \& Villarroel (2008), no siempre es merecida. Bajo el objetivo de poner en marcha aulas digitales del siglo XXI, con una dotación de infraestructuras y conectividad acordes a éste, surge el programa Escuela 2.0.

Cuando hablamos de la Escuela TIC 2.0, nos referimos a la versión andaluza del programa estatal Escuela 2.0. Éste se presenta en el debate del Estado de la Nación del 12 de mayo de 2009, como una iniciativa para la innovación y la modernización de los sistemas de enseñanza. De esta forma, se da comienzo a un proyecto de grandes pretensiones que Pérez (2011) califica como un proyecto ambicioso y acorde a los ciudadanos del siglo XXI.

Para este proyecto, el gobierno dota a las aulas de los elementos necesarios, dentro y fuera de ellas y se propone formar al profesorado adecuadamente. Se aclara que la iniciativa se llevará a cabo en colaboración con todas las comunidades autónomas a partir del curso 2009/10. Si bien es cierto que la predisposición del Gobierno central es hacer extensible dicho proyecto a todas las comunidades, no todas ponen en funcionamiento el mismo (Madrid o Valencia) y tampoco todas adoptan las líneas fundamentales de actuación (Baleares, Cataluña o Galicia).

En el caso de la comunidad autónoma andaluza, el programa se adapta con total fidelidad al diseño original, firmándose el convenio de colaboración entre el Ministerio de Educación y el Gobierno andaluz en octubre de 2009. Ambas partes se comprometen a cofinanciar el programa a partes iguales.

Así pues, cuando el Gobierno andaluz lanza el programa, indica que cada estudiante de $5^{\circ}$ y $6^{\circ}$ de Primaria y $1^{\circ}$ y $2^{\circ}$ de ESO dispondrá de un ordenador portátil y cada centro público estará dotado de aulas con pizarra digital, cañón de proyección y equipo multimedia, portátiles para uso del equipo docente, conexión WI-FI dentro del aula, 
conexión a internet del centro a través de la Red Corporativa de la Junta de Andalucía y mueble para la alimentación de las baterías. Además, la Junta de Andalucía pone al servicio de la comunidad educativa, diferentes manuales para el buen uso de éstas, una mochila digital con recursos digitales, un servicio para el mantenimiento de los equipos $\mathrm{y}$ folletos informativos para las familias.

En lo que a la formación del profesorado se refiere, la Comunidad Autónoma andaluza suscribe con el Ministerio de Educación el compromiso de formación fijado, ideando un modelo formativo propio, complementario al del INTEF (Instituto Nacional de Tecnologías Educativas y de Formación del Profesorado). Este programa de formación, puesto en marcha durante el curso 2010-11, está planificado en tres módulos flexibles que pretenden adaptarse al nivel de competencia digital del profesorado: formación básica para el uso del equipamiento 2.0, aplicaciones prácticas de las aulas $2.0 \mathrm{y}$ otros recursos formativos TIC.

En definitiva, con esta dotación tecnológica, formación del profesorado, recursos TIC y propaganda informativa para las familias, se conforman las dimensiones de la Escuela TIC 2.0. Sin embargo, a pesar de las sucesivas adendas concedidas para el mantenimiento financiero de este programa, en abril de 2012, la Secretaría de Estado de Educación, Formación Profesional y Universidades anunciaba, en los ajustes presupuestarios de 2012, la supresión del programa Escuela 2.0 por no adecuarse a los principios de eficiencia, austeridad y priorización del gasto que lideraban dichos ajustes. Anunciaba también que este programa sería sustituido por otro, del que sólo se esbozaban las líneas generales.

Por su parte, la Comunidad Autónoma andaluza comenzaba el curso escolar 201213 sin uno de sus referentes, la Escuela TIC 2.0. En octubre de 2012, saltaba la noticia de que la Junta cambiaría los portátiles de uso personal por equipamiento para el colegio (Rincón, 2012). El Gobierno andaluz se comprometía a enviar en breve un paquete con un número determinado de portátiles dependiendo de las unidades de $5^{\circ}$ de Primaria del centro. En abril de 2014, y sin haberse instaurado la nueva ley educativa, el Gobierno andaluz propone una medida para la introducción de las Tecnologías de la Información y la Comunicación, se trata esta vez de dotar al alumnado de $6^{\circ}$ de Primaria de tabletas híbridas.

Es destacable que, a pesar del amplio despliegue y repercusión social de este programa, no hayamos encontrado investigaciones al respecto. Pocos son también los estudios (Almerich, Suárez, Orellana, Belloch, Bo \& Gastaldo, 2005; Cox, Preston \& Cox, 1999; Kubiatko, 2013; Morris \& Venkatesh, 2000; Saleh \& Sa'ad, 2013; Torres, Robles \& Molina, 2011) acerca de la influencia que tiene la edad sobre las decisiones para la adopción de la tecnología. En dichas investigaciones, focalizadas en estudios de caso diversos, se apunta la misma idea, es decir, la importancia que tiene la edad en la integración de las TIC en el proceso de enseñanza-aprendizaje, así como que el uso y actitudes del profesorado hacia éstas será distinto en función de la edad.

La teoría más extendida sobre las razones por las que algunas personas utilizan las tecnologías y sus actitudes hacia las mismas es la etiquetada como Technological Acceptance Model, diseñada por Davis, Bagozzi \& Warshaw (1989). Esta teoría indica 
que la actitud, y por consiguiente el uso de las TIC, se relaciona con la utilidad percibida, la facilidad de uso percibida y los lazos psicológicos del ciudadano.

Pese a la enorme validez y aceptación de este modelo, es interesante considerar otros factores que influyen en la actitud que puede determinar el uso o no de las TIC. Por un lado, podríamos tener en cuenta el papel que desempeñan las infraestructuras tecnológicas, en cuanto que éstas condicionarían la facilidad de uso de las TIC; y por otro, la influencia que variables sociales como la edad, género, experiencia y voluntariedad tienen sobre la actitud hacia éstas (Torres et al., 2011).

Centrándonos en la edad, son varios los estudios que amparan la idea de que este factor incide directamente sobre el uso de las TIC (Kubiatko, 2013; Morris \& Venkatesh, 2000; Torres et al., 2011). Otros estudios, aunque revelan un posible impacto del factor edad, lo consideran inferior al que puedan tener otros aspectos como es el género (Almerich et al., 2005; Saleh \& Sa’ad, 2013).

\section{Metodología}

El objetivo de esta investigación es conocer la relación entre la edad del profesorado de música de la ESO andaluz y el desarrollo del programa Escuela TIC 2.0., definida por cinco dimensiones, que son: la presencia de las tecnologías en el aula, la formación del profesorado y por ende su actitud hacia las TIC, los recursos digitales de los que se acompaña el programa y la proyección del mismo en las familias andaluzas.

La muestra que participó en el estudio, fue calculada aplicando el muestreo probabilístico aleatorio simple de Tagliacarne (1968). La muestra es representativa y estuvo conformada por 207 docentes de música pertenecientes a centros públicos de Andalucía de enseñanza secundaria, siendo la población total de 860 docentes.

Un 50,7 \% eran mujeres y un $49,3 \%$ hombres. La mayor parte de los participantes se caracterizaba por tener entre 35 y 44 años $(50,2 \%)$, seguidos por aquellos que oscilaban entre 45 y 54 años $(27,1 \%)$ y los que iban de los 25 a los 34 años $(17,9 \%)$. Por lo que respecta al origen del profesorado por provincias, la mayor participación proviene de Sevilla (24,2\%), Córdoba (15\%) y Málaga (12,6\%). Sigue Cádiz y Granada con igual porcentaje $(11,6 \%)$ y finalmente, Almería $(9,2 \%)$, Jaén y Huelva $(7,7 \%)$.

En relación a la formación inicial en nuevas tecnologías, el 58,8\% expresa su inexistencia. Sobre la experiencia docente del profesorado de música, hay que señalar que el $34,3 \%$ se ubicaba entre los 11 y 15 años. Similar porcentaje hallado en los que habían trabajado entre 6 y más de 20 años y un 2,9\%, inferior a 5 años. El 89,4 \% del personal encuestado era funcionario con destino definitivo en su centro.

Para llevar a cabo nuestra investigación, empleamos el método descriptivo (Cohen \& Manion, 1990) y para la recogida de datos, el instrumento en el que nos basamos fue un cuestionario, en cuanto que, como señala McMillan \& Schumacher (2005), nos permite transformar un conjunto de números u observaciones en índices que describen 
o caracterizan los datos, resumiendo, organizando y reduciendo grandes cantidades de observaciones.

El cuestionario, de diseño propio (ver anexo 1), se estructuraba en siete bloques y 27 ítems. El primer bloque, estaba dedicado a los datos identificativos de los encuestados referidos al sexo, a la experiencia docente, al tipo de centro, a la provincia en la que se impartía docencia o a la categoría profesional (ítems 1 al 6). En cuanto al segundo, referido a la formación académica general del profesorado, tanto inicial, como de asignaturas cursadas sobre nuevas tecnologías, se emplearon ítems de respuesta múltiple, dejándose un espacio abierto ante la posibilidad de que ninguna de las opciones ofertadas fuese válida para los encuestados (ítems 7 y 8). Por lo que respecta al resto de los bloques, se utilizó una escala Likert que respondía a las categorías nada 1 , poco 2 , suficiente 3 , bastante 4 y mucho 5 . A estos últimos, se les denominó con las dimensiones que definían al programa Escuela TIC 2.0, esto es, la formación en nuevas tecnologías (dimensión III, ítems 9 al 12), las tecnologías en el aula (dimensión IV, ítems 13 al 17), la actitud hacia las TIC y las competencias (dimensión V, ítems 18 al 21), los recursos TIC (dimensión VI, ítems 22 al 25), la vinculación entre la Escuela TIC 2.0 y las familias (dimensión VII, ítem 26) y otros comentarios (ítem 27).

Para la construcción del cuestionario llevamos a cabo no sólo una exhaustiva revisión teórica en torno a la Escuela 2.0 y a la integración de las TIC en educación, sino que partimos de la participación y coordinación de foros de debate relacionados con la misma temática, instrumento éste, que también ayudó a configurar el mismo.

En lo que se refiere a la recogida de la información, utilizamos una aplicación web gratuita de código abierto llamada Limesurvey, alojada en un servidor de la Universidad de Granada. A través de dicha aplicación, el cuestionario fue enviado a todos los centros de enseñanza secundaria de Andalucía, acompañándose de una carta de presentación acerca del estudio. El sistema empleado nos permitía controlar el estado de la encuesta, ofreciéndonos la opción de volver a enviar el cuestionario solamente a aquellos centros cuyo profesorado de música no hubiesen cumplimentado el mismo.

Para determinar la validez del cuestionario, recurrimos a la validación por jueces y a la validación estadística. En cuanto a los jueces, 14, se ocuparon de interpretar, a través de un formulario, el significado de los diferentes ítems que lo componían así como de valorar su claridad, pertinencia y relevancia con una ponderación de 1 (mínimo) a 5 (máximo). Dicha revisión fue llevada a cabo por un conjunto de expertos en el ámbito investigador y en el musical: 9 pertenecían al departamento de Didáctica de la Expresión Musical de las Universidades de Granada, Sevilla, Cantabria, Complutense de Madrid y Castilla-La-Mancha; 3 de Didáctica y Organización Escolar de Málaga y Granada; 1 de Métodos de Investigación y Diagnóstico en Educación de Granada; y 1 del Real Conservatorio Superior de Música de Madrid. Su valoración fue muy positiva, haciendo éstos, en la mayoría de los casos, indicaciones formales y tipográficas, las cuales se consideraron, desestimándose aquellas fundamentadas en juicios de valor sobre la temática en cuestión y no sobre el cuestionario en sí mismo. 
La validación estadística se realizó mediante el análisis de componentes principales. Este nos permitió detallar la estructura de las interrelaciones, calculando factores explicativos de dichas interrelaciones (Rodríguez, Olmos \& Martínez, 2012). Para medir el grado de correlación significativa entre la variable empírica (o indicador) y el factor (o variable latente) utilizamos el estadístico factor loading, que usualmente ha de ser superior a .30, aunque en nuestro caso partimos de .40 (Cea, 2004). Nuestro análisis nos ofreció un valor KMO de adecuación muestral de 0.611 , que indicaba un grado de intercorrelación aceptable. La prueba de esfericidad de Barlett nos dio un valor de $p=.000$. Por lo tanto, entendimos que la información obtenida de la muestra se distribuyó de manera adecuada para llevar a cabo dicho análisis.

Determinados cinco factores al concluir el análisis, se pudo constatar que tres de ellos se adecuaban a tres de los bloques que conformaban nuestro cuestionario (ver tabla 1).

\section{FACTORES BLOQUES DEL CUESTIONARIO \\ I. Utilización y repercusión de las TIC en el V. Competencias en TIC proceso de enseñanza-aprendizaje. \\ II. Formación en nuevas tecnologías \\ IV. Tecnologías en el aula \\ III. Formación en nuevas tecnologías \\ IV. Tecnologías en el aula}

Tabla 1. Adecuación entre los factores y los bloques del cuestionario

Para determinar la fiabilidad del cuestionario, se siguió a Bisquerra (1987) y se recurrió a dos análisis: $\alpha$ de Cronbach y Dos mitades. Al aplicar el primer análisis obtuvimos un coeficiente de .967, lo cual implicaba una alta fiabilidad. En efecto, McMillan \& Schumacher (2005) consideran una fiabilidad satisfactoria coeficientes superiores a .70. Al realizar Dos mitades, la correlación para ambas partes de Spearman-Brown fue de ,787. La consistencia interna para cada una de las dos mitades fue de ,935 para la primera parte y de ,958 para la segunda. Siguiendo la misma teoría, la fiabilidad del instrumento utilizado fue satisfactoria.

\section{Análisis de los Resultados}

El objetivo pretendido en este estudio fue determinar si existía una asociación significativa entre la edad del profesorado de música de la ESO y el desarrollo de la Escuela TIC 2.0. Hemos aplicado el análisis de contingencias, que ha sido desarrollado empleando el programa IBM SPSS Statistics 20.

El desarrollo de la Escuela TIC 2.0 contemplaba actuaciones, como así recogía nuestro cuestionario, referidas a la formación en tecnologías, a la presencia de tecnología en el aula, a las actitudes del profesorado, a los recursos y a la familia, de modo que abordamos el tipo de asociación que existía entre cada una de estas con la edad. 
La primera asociación significativa en la formación la encontramos con el manejo del correo electrónico (ver tabla 2). La mayoría del profesorado afirmaba tener bastante (opción 4) o mucho (5) conocimiento. El porcentaje era mayor en la opción 5 conforme disminuía la franja de edad, es decir, cuanto más joven era el profesorado, mayor era el conocimiento que se tenía del correo.

En relación a la sindicación de contenidos, la tendencia era la de admitir poseer un conocimiento bastante bajo del uso de dicha aplicación y a medida que aumentaba el rango de edad, esta tendencia se agudizaba. A partir de 45 años, el profesorado que afirmaba tener poco o nada de conocimiento superaba el 50\%. Por debajo de los 45 años, los grupos rondaban estas mismas cifras.

\begin{tabular}{|c|c|c|c|c|c|c|c|c|c|}
\hline \multicolumn{8}{|c|}{ Conocimiento que posee sobre el manejo del correo electrónico } & \multirow[t]{3}{*}{$\chi^{2}$} & \multirow[t]{2}{*}{$\mathrm{p}$} \\
\hline & & \multicolumn{6}{|c|}{$\%$} & & \\
\hline & & 1 & 2 & 3 & 4 & 5 & Total & & \multirow{5}{*}{0,029} \\
\hline \multirow[t]{4}{*}{ Edad } & $25-34$ & 3,2 & 0 & 0 & 15,6 & 81,2 & 100 & \multirow{4}{*}{18,580} & \\
\hline & $35-44$ & 0 & 0 & 5,1 & 20,7 & 74,2 & 100 & & \\
\hline & $45-55$ & 0 & 0 & 8 & 28 & 64 & 100 & & \\
\hline & +55 & 12,5 & 0 & 12,5 & 12,5 & 62,5 & 100 & & \\
\hline
\end{tabular}

Conocimiento que posee sobre el manejo de la sindicación de contenidos (ej. Google reader)

\begin{tabular}{|c|c|c|c|c|c|c|c|c|c|}
\hline \multirow[t]{4}{*}{ Edad } & $25-34$ & 12,5 & 37,5 & 9,4 & 21,8 & 18,8 & 100 & \multirow{3}{*}{28,812} & \\
\hline & $35-44$ & 32,3 & 14,6 & 25 & 10,6 & 11,5 & 100 & & \\
\hline & $45-55$ & 30 & 30 & 28 & 4 & 8 & 100 & & \\
\hline & +55 & 62,5 & 10,5 & 0 & 0 & 25 & 100 & & \\
\hline
\end{tabular}

Tabla 2. Edad y Formación en Tecnología. Leyenda: 1 nada, 2 poco, 3 suficiente, 4 bastante, 5 mucho.

En la tabla 3, mostramos la asociación entre la edad y la dimensión tecnología del aula. Fue unánime la valoración positiva de contar con un proyector en el aula de música. En todos los grupos de edades la tendencia era que todos lo consideraban fundamentalmente muy bueno, pero en especial, el grupo entre 35 y 44 años. Cuando abordamos el uso del proyector en la actividad docente, destacamos cómo el profesorado comprendido entre los 35 y los 44 años, era el que declaraba hacer un mayor uso. Sobre el uso de las tecnologías para cantar en el karaoke (tabla 3), los que afirmaban usarlas menos eran los mayores de 55 años, mientras que el profesorado entre 45-54 años eran quienes más las utilizaban, aunque no superaban el 50\%. 


\begin{tabular}{|c|c|c|c|c|c|c|c|c|c|}
\hline \multicolumn{8}{|c|}{ Señale en qué medida considera necesario contar con el proyector } & $\chi^{2}$ & $\mathrm{p}$ \\
\hline & & $\%$ & & & & & & \multirow{6}{*}{34,113} & \multirow{6}{*}{0,001} \\
\hline & & 1 & 2 & 3 & 4 & 5 & Total & & \\
\hline \multirow[t]{4}{*}{ Edad } & $25-34$ & 0 & 0 & 12,9 & 16,1 & 71 & 100 & & \\
\hline & $35-44$ & 0 & 1,1 & 6,4 & 10,8 & 81,7 & 100 & & \\
\hline & $45-55$ & 10,4 & 4,3 & 6,2 & 6,2 & 72,9 & 100 & & \\
\hline & +55 & 0 & 0 & 14,3 & 57,1 & 28,6 & 100 & & \\
\hline
\end{tabular}

En su actividad docente, señale el grado de utilización que hace del proyector

\begin{tabular}{llllllll}
\hline Edad & $25-34$ & 6,5 & 3,2 & 12,9 & 12,9 & 64,5 & 100 \\
\cline { 2 - 7 } & $35-44$ & 6,5 & 3,2 & 4,2 & 15,1 & 71 & 100 \\
\cline { 2 - 7 } & $45-55$ & 22,9 & 2,1 & 8,3 & 14,6 & 52,1 & 100 \\
\cline { 2 - 7 } & +55 & 14,3 & 0 & 42,9 & 28,6 & 14,2 & 100 \\
\hline
\end{tabular}

En referencia a su actividad docente, indique el grado en el que utiliza las TIC para cantar en karaoke

\begin{tabular}{cccccccc}
\hline Edad & $25-34$ & 19,4 & 38,6 & 19,4 & 16,1 & 6,5 & 100 \\
\cline { 2 - 8 } & $35-44$ & 29,3 & 14,2 & 12 & 15,2 & 29,3 & 100 \\
\cline { 2 - 8 } & $45-55$ & 31,2 & 4,3 & 20,8 & 22,9 & 20,8 & 100 \\
\cline { 2 - 8 } & +55 & 57,1 & 14,3 & 14,3 & 57,1 & 14,2 & 100
\end{tabular}

Tabla 3. Edad y Tecnología en el aula.

En cuanto a las actitudes y la edad (tabla 4), los datos mostraron que los que más se inclinaban a favor de las metodologías tradicionales eran los mayores de 55 años. Parecía, por los datos, que a menor edad, mayor era la resistencia hacia las metodologías tradicionales y viceversa. Existía relación entre la edad y la actitud hacia el uso de las TIC, como se aprecia en la tabla, bajo la creencia de que suponía un aumento de la calidad del aprendizaje del estudiante. Los que más apostaron por ello eran los más jóvenes, con un 32,1\% que lo valoraban como muy bueno y un 46,4\% bastante bueno.

\begin{tabular}{|c|c|c|c|c|c|c|c|c|c|}
\hline \multicolumn{8}{|c|}{$\begin{array}{l}\text { Valore el obstáculo que conlleva las TIC en el aula: aunque tengo la } \\
\text { preparación suficiente para su uso, prefiero utilizar metodologías } \\
\text { tradicionales }\end{array}$} & \multirow{2}{*}{$\chi^{2}$} & \multirow{2}{*}{$\begin{array}{c}\mathrm{p} \\
0,006\end{array}$} \\
\hline & & \multicolumn{6}{|c|}{$\%$} & & \\
\hline & & 1 & 2 & 3 & 4 & 5 & Total & & \\
\hline \multirow[t]{4}{*}{ Edad } & $25-34$ & 44,8 & 24,1 & 17,4 & 10,3 & 3,4 & 100 & & \\
\hline & $35-44$ & 31,6 & 27,8 & 29,2 & 3,8 & 7,6 & 100 & & \\
\hline & $45-55$ & 31,9 & 23,4 & 40,4 & 4,3 & 0 & 100 & & \\
\hline & +55 & 0 & 14,2 & 42,9 & 42,9 & 0 & 100 & & \\
\hline
\end{tabular}




\begin{tabular}{|c|c|c|c|c|c|c|c|c|c|}
\hline \multicolumn{8}{|c|}{$\begin{array}{l}\text { Valore sus actuaciones propias ante las TIC: Integro las TIC en la } \\
\text { docencia, porque incrementa la calidad de los aprendizajes de los } \\
\text { estudiantes }\end{array}$} & \multirow[t]{5}{*}{22,508} & \multirow[t]{5}{*}{0,032} \\
\hline \multirow[t]{4}{*}{ Edad } & $25-34$ & 3,6 & 7,1 & 10,7 & 46,4 & 32,2 & 100 & & \\
\hline & $35-44$ & 3,8 & 7,6 & 26,6 & 36,7 & 25,3 & 100 & & \\
\hline & $45-55$ & 0 & 17 & 31,9 & 29,8 & 21,3 & 100 & & \\
\hline & +55 & 0 & 0 & 85,7 & 0 & 14,3 & 100 & & \\
\hline
\end{tabular}

Tabla 4. Edad y Actitud hacia las TIC y Competencias.

En torno a los recursos TIC, a menor edad, mejor era la valoración que se hacía del uso de otras webs en el aula. Los mayores de 55 años se situaban en un 83,3\% en torno a los valores nada y poco (tabla 5).

\begin{tabular}{|c|c|c|c|c|c|c|c|c|c|}
\hline \multicolumn{8}{|c|}{ Valore el uso que hace de otras webs en el aula } & \multirow{3}{*}{$\begin{array}{l}\chi^{2} \\
21,676\end{array}$} & \multirow{2}{*}{$\begin{array}{l}p \\
0,041\end{array}$} \\
\hline & & \multicolumn{6}{|c|}{$\%$} & & \\
\hline & & 1 & 2 & 3 & 4 & 5 & Total & & \\
\hline \multirow[t]{4}{*}{ Edad } & $25-34$ & 14,3 & 10,7 & 7,1 & 32,2 & 35,7 & 100 & & \\
\hline & $35-44$ & 7,9 & 5,2 & 23,7 & 30,3 & 32,9 & 100 & & \\
\hline & $45-55$ & 18,2 & 9,1 & 22,7 & 25 & 25 & 100 & & \\
\hline & +55 & 50 & 33,3 & 16,7 & 0 & 0 & 100 & & \\
\hline
\end{tabular}

Tabla 5. Edad y Recursos TIC.

Al comparar la edad con la familia (tabla 6), era unánime la idea del profesorado respecto a que el alumnado no recibía la supervisión de los padres cuando navegaban por internet. Todos los grupos se ubicaban en los valores nada y poco.

\begin{tabular}{|c|c|c|c|c|c|c|c|c|c|}
\hline \multicolumn{8}{|c|}{ Los estudiantes son supervisados por los padres al utilizar internet } & $\chi^{2}$ & $p$ \\
\hline & & \multicolumn{6}{|c|}{$\%$} & \multirow[t]{6}{*}{17,332} & \multirow[t]{6}{*}{0,044} \\
\hline & & 1 & 2 & 3 & 4 & 5 & Total & & \\
\hline \multirow[t]{4}{*}{ Edad } & $25-34$ & 42,9 & 39,2 & 17,9 & 0 & 0 & 100 & & \\
\hline & $35-44$ & 30,1 & 52,1 & 16,4 & 1,4 & 0 & 100 & & \\
\hline & $45-55$ & 28,6 & 61,9 & 9,5 & 0 & 0 & 100 & & \\
\hline & +55 & 50 & 33,3 & 0 & 16,7 & 0 & 100 & & \\
\hline
\end{tabular}

Tabla 6. Edad y Escuela TIC 2.0 y Familia.

\section{Discusión y Conclusiones}

El objetivo de este estudio es conocer la relación entre la edad del profesorado de música de la ESO andaluz y el desarrollo del programa Escuela TIC 2.0., definido por 
cinco dimensiones, que son la formación en tecnología, la presencia de tecnologías en el aula, las actitudes, los recursos y la familia.

En base a los resultados anteriores, podemos concluir que a medida que el profesorado de música es mayor, es también más reticente a incorporar las nuevas tecnologías en el aula. De igual forma, cuanto más joven es este colectivo, más proclive se muestra a utilizarlas.

En relación a la primera dimensión, la formación, cuando abordamos el manejo del correo electrónico observamos cómo se ha integrado en la práctica habitual del profesorado. No obstante, se ve claramente que las personas de mayor rango de edad, encuentran una mayor dificultad a la hora de darle funcionalidad al mismo. Si bien es cierto que el correo electrónico es uno de los servicios más utilizados por las personas mayores (Herrero \& García, 2007; Martínez-Pecino, García, Guerra y Troya, 2009), autores como De Oleo (2010) apuntan hacia el aumento de problemas de usabilidad de las TIC con la edad.

La sindicación de contenidos no parece ser una herramienta demasiado integrada en los hábitos de uso del profesorado de música andaluz, aunque el empleo y el conocimiento de ésta son elevados en los profesionales de edad más temprana. Desconocemos las razones por las que no se integra en la práctica docente, lo cual choca con las ideas de Franganillo \& Catalán (2005), quienes afirman que la sindicación de contenidos puede suponer una auténtica revolución en los entornos virtuales de aprendizaje, ya que los contenidos se recuperan, reutilizan y actualizan cómodamente.

Sobre la segunda dimensión, la presencia de tecnologías en el aula, todos los grupos consideran muy necesario contar con un proyector en el aula de música. La clave es si lo usan y cómo, ya que según señala Saleh \& Sa'ad (2013) la instalación de las tecnologías en las aulas no es suficiente para llevar a cabo una integración real, donde aspectos como el tipo de dotación, su localización, mantenimiento, factores de índole pedagógica y personal, son también muy importantes. En este punto, si bien es cierto que se prioriza la dotación tecnológica de los centros y pese a modernizar tecnológicamente los mismos, se continúa anclado en paradigmas transmisores (Aparici, 2001). No obstante, lo que está claro es que la Escuela TIC 2.0, mediante la pizarra digital, ofrece la posibilidad de convertir ésta en un proyector, de ahí que podamos concluir que el programa satisface una necesidad unánime en el profesorado siendo el grupo más joven, el que más valora contar con este tipo de infraestructura tecnológica.

Frente a la necesidad de contar con un proyector, cuando valoramos el uso que se hace del mismo, nos encontramos un rango de porcentajes más bajo en todos los grupos. Lo cual pone de manifiesto que, aunque la creen una herramienta positiva para la práctica educativa, luego no lo incorporan del mismo modo a su actividad docente. En líneas generales, encontramos un profesorado que pese a tener un gran deseo por integrar las TIC en la educación, encuentra también muchos obstáculos, entre los cuales se han señalado como significativos: la falta de confianza, la falta de competencia y la falta de acceso a los recursos (Bingimlas, 2009). No obstante, entre 
los grupos de edad más jóvenes su uso es bastante alto, tendencia que decrece en cuanto asciende la edad.

Parece también existir una relación directa entre la edad y el uso de las metodologías tradicionales, pese a poseer la preparación necesaria para impartir la docencia con otro planteamiento didáctico. De nuestro análisis, se colige que a medida que el profesorado es más mayor, es también más proclive a usar las metodologías tradicionales y a desdeñar el uso y la incorporación de tecnologías. En efecto, la integración de las TIC en el aula modifica nuestra forma de enseñar, lo cual genera en los docentes desconfianza y rechazo en cuanto que éstos están acostumbrados a métodos que durante mucho tiempo les han dado resultados óptimos (Romero, 2004). En contraposición, de nuestro estudio también se desprende, que son los más jóvenes los que mejor valoración tienen sobre la capacidad de las TIC para incrementar la calidad de los aprendizajes de los estudiantes.

Otra dimensión clave para incorporar las tecnologías es la actitud, en cuanto que, dependiendo de la creencia y la percepción que se tenga de las mismas, se van a utilizar o no (Gargallo, Suárez \& Almerich, 2006; Ramírez, Cañedo \& Clemente, 2012). Prensky (2001) diferenciaba entre nativos e inmigrantes digitales y ponía de manifiesto una contraposición de actitudes. Si trasladásemos esta diferenciación a nuestro estudio el profesorado de música más longevo podría estar dentro de ese grupo de inmigrantes que suelen inquietarse ante las TIC, mostrar su desconfianza, y con frecuencia, rechazarlas; mientras que el profesorado más joven, se mostraría más cercano al perfil del nativo, conocedor y amigo de esta nueva lengua.

Cuando valoramos la cuarta dimensión, los recursos, y más concretamente el uso que se hace de otras webs en el aula, nos encontramos una situación que se viene ya repitiendo en todo el estudio. Los que mayor valoración conceden al acceso de este tipo de herramientas son los más jóvenes, con un uso por encima de la media, sin embargo, este va decreciendo conforme se avanza en edad. La falta de acceso a los recursos, según BECTA, citado por Bingimlas (2009), puede responder a la pobre organización de los recursos, escasa calidad del hardware, inapropiado software o inapetencia del profesorado. Ésta última estaría relacionada con la escasez de formación, y por tanto, carencia de habilidades pedagógicas para integrar las TIC en su práctica docente, lo cual generaría una baja autoestima y confianza en el profesorado en relación a estos medios que puede derivar en la no integración de recursos.

Finalmente, en la última dimensión, hay un claro consenso, por parte del profesorado, en la idea de que el alumnado no recibe la supervisión de sus padres a la hora de utilizar internet. Afirmación que se contrapone al estudio de Livingstone y Haddon (2009), citados por Martínez, Cortés, Medrano \& Apocada (2014) quienes señalan que la mediación parental en España es muy elevada, encontrándose incluso entre los niveles más altos de Europa. Sin embargo, también es cierto que se viene poniendo en práctica un tipo de mediación restrictiva, esto es, un tipo de supervisión y vigilancia caracterizados por el establecimiento de normas como medida para controlar el uso de internet (Shin, 2014). Si bien Livingstone y Helsper (2008) consideran que la mediación basada en la restricción reduce los riesgos del uso de internet, otros autores, apuestan por una mediación activa (Shin, 2014) también denominada instructiva 
(Martínez et al., 2014) en cuanto que implica una mayor participación por parte de los padres con sus hijos y supone también una discusión e instrucción al respecto. Evidentemente, en nuestro estudio el objetivo es conocer la opinión que tiene el profesorado al respecto y no descubrir la práctica real, que abriría otra línea de investigación.

Teniendo en cuenta que asistimos a un nuevo cambio legislativo en educación con la implantación de la ley Orgánica para la Mejora de la Calidad Educativa (LOMCE), que conllevará una nueva política en materia de tecnología educativa, consideramos que este estudio ha de tenerse en cuenta a la hora de establecerse medidas tanto en inversiones tecnológicas, creación de recursos TIC, diseño de itinerarios formativos, así como planteamientos de interacción entre las familias y la institución educativa. No obstante, consideramos que sería muy interesante completar este estudio con una investigación cualitativa, configurando para ello grupos de discusión en los que participasen representantes de las diferentes partes implicadas en dicho programa. Siendo conscientes de la gran limitación que supone la inexistencia de un marco teórico específico sobre el profesorado de música en la etapa de enseñanza secundaria en relación a la Escuela TIC 2.0.

En definitiva, y tras lo arrojado en este estudio, podemos concluir que la edad de los docentes de música de la ESO se asocia a la incorporación y utilización de las TIC. Y por ende, al desarrollo de programas como la Escuela TIC 2.0 en sus diferentes dimensiones: formación en tecnologías, presencia de tecnología en el aula, actitudes del profesorado, recursos y vinculación entre la familia y el centro educativo.

\section{Referencias bibliográficas}

ALMERICH, G., SUÁREZ, J.M., ORELlANA, N., BELLOCH, C., BO, R. \& GASTALDO, I. (2005). Diferencias en los conocimientos de los recursos tecnológicos en profesores a partir del género, edad y tipo de centro. Relieve, 11 (2), 127-146.

APARICI, R. (2011). Principios pedagógicos y comunicacionales de la Educación 2.0. La educación, revista digital, 145. Recuperado de http://goo.gl/NmoCIp, 14 de mayo 2014.

BRITISH EDUCATIONAL COMMUNICATIONS AND TECHNOLOY AGENCY (BECTA) (2004). Review of the research literature on barriers to the uptake of ICT by teachers. Recuperado el 13 de agosto de 2008 de http://www.becta.org.uk

BINGIMLAS, K.A. (2009). Barriers to the Successful Integration of ICT in Teaching and Learning Environments: A Review of the Literature. Eurasia Journal of Mathematics, Science \& Technology Education, 5 (3), 235-245.

BISQUERRA, R. (1987). Introducción a la estadística aplicada a la investigación educativa. Un enfoque informático con los paquetes BMDP y SPSS. Barcelona: PPU.

CASTAÑO, C., PALACIO, G., MAÍZ, I. \& VILlARROEL, J. (2008). Prácticas educativas en entornos web 2.0. Madrid: Síntesis. 
CEA, M.A. (2004). Métodos de encuesta. Teoría y práctica, errores y mejora. Madrid: Síntesis.

COHEN, L. \& MANION, L. (1990). Métodos de investigación educativa. Madrid: La Muralla.

COX, M., PRESTON, C. \& COX, K. (1999). What factors support or prevent teachers fromusing ICT in their classrooms? A paper presented at British Educational Research Association Annual Conference, University of Sussex at Brighton. Recuperado de http://goo.gl/eVxAlN, 1 de mayo de 2012.

DE OLEO, C. (2010). La usabilidad y la edad. Sociedad y Utopía: Revista de Ciencias Sociales, 36, 235-246.

DAVIS, F.D, BAGOZZI, R.P. \& WARSHAW, P.R. (1989). User acceptance of computer technology: a comparison of two theoretical models. Management Science, 35 (8), 982-1003.

FRANGANILLO, J. \& CATALÁN, M.A. (2005). Bitácoras y sindicación de contenidos: dos herramientas para difundir información. BiD: textos universitaris de biblioteconomia i documentació, 15. Recuperado de http://goo.gl/YsQwsm, 18 de mayo de 2014.

GARGALlO, B., SUÁREZ, J. \& ALMERICH, G. (2006). La influencia de las actitudes de los profesores en el uso de las nuevas tecnologías. Revista Española de Pedagogía, 64 (233), 45-66.

Herrero, J. \& García, E. (2007). Las personas mayores internautas: quiénes son, qué hacen cuando se conectan y qué buscan en Internet. XII Congreso Internacional Mundo Internet. Recuperado de http://goo.gl/N794Tc, 10 de octubre de 2013.

KUBIATKO, M. (2013). The comparison of different age groups on the attitudes towards and the use of ICT. Educational Sciences: Theory \& Practice, 13 (2), 12631272.

LEY ORGÁNICA 8/2013, de 9 de diciembre, para la mejora de la calidad educativa. B.O.E. n 295 (10-12-2013).

LIVINGSTONE, S. \& HADDON, L. (2009). EU Kids Online: Final Report. LSE, London: EU Kids Online (EC Safer Internet Plus Programme Deliverable D6.5). Recuperado de http://goo.gl/k7RYQY, 20 de mayo de 2014.

LIVINGSTONE, S. \& HELSPER, E. (2008). Parental mediation and children's Internet use. Journal of Broad Casting \& Electronic Media, 52 (4), 581-599.

MARTínEZ, J.A., CORTÉS, A., MEDRANO, C. \& APODACA, P. (2014). Internet use and parental mediation: A cross-cultural study. Computers \& Education, 70, 212-221. . (doi: http://dx.doi.org/10.1016/j.compedu.2013.07.036).

MARTÍNEZ-PECINO, R., GARCÍA, A. J., GUERRA, J. M., \& TROYA, Y. (2009). La comunicación en personas mayores mediante internet y el móvil. IV Congreso de la Cibersociedad. Recuperado de http://goo.gl/rcuzGA, 6 de abril de 2014. 
MCMILLAN, J.H. \& SCHUMACHER, S. (2005). Investigación educativa (5 $5^{\mathrm{a}}$ ). Madrid: Pearson.

MORRIS, M. G. \& VENKATESH, V. (2000). Age differences in technology adoption decisions. Personnel Psychology, 53, 375-403. (doi: 10.1111/j.17446570.2000.tb00206.x).

PÉREZ, A. (2011). Escuela 2.0. Recuperado de http://goo.gl/WvU02, 29 de diciembre de 2011.

PRENSKY, M. (2001). Digital Natives, Digital Inmigrants. On the horizon, 9 (5), 1-6. (doi: http://dx.doi.org/10.1108/10748120110424816).

RAMÍREZ, E., CAÑEDO, I. \& CLEMENTE, M. (2012). Las actitudes y creencias de los profesores de Secundaria sobre el uso de internet en sus clases. Comunicar, 38 (19),147-155. (doi: http://dx.doi.org/10.3916/C38-2012-03-06).

RINCÓN, R. (2012, 8 de octubre), Educación entregará 27500 ordenadores de uso colectivo en los centros públicos. El País. Recuperado de http://goo.gl/y6ctT, 2 de enero de 2013.

ROMERO, J.B. (2004). Las nuevas tecnologías y la expresión musical, otros lenguajes en la educación. Comunicar. Revista Científica de Comunicación y Educación, 23, 25-30.

RODRÍGUEZ, M.J, OLMOS, S. \& MARTÍNEZ, F. (2012). Propiedades métricas y estructura dimensional de la adaptación española de una escala de evaluación de competencia informacional autopercibida (IL-HUMASS). Revista de Investigación Educativa, 30 (2), 347-366. .

SALEH, H. \& SA'AD, A. (2013).The Impact of Teachers' Age, Gender and Experience on the Use of Information and Communication Technology in EFL Teaching. English Language Teaching, 6 (6), 57-67. (doi: http://dx.doi.org/10.5539/elt.v6n6p57).

SHIN, W. (2013). Parental socialization of children's Internet use: A qualitative approach. New Media \& Society, 15 (8), 1-17. (doi: 10.1177/1461444813516833).

TAGLIACARNE, G. (1968). Técnica y práctica de la investigación de mercado. Barcelona: Ariel.

TORRES, C., ROBLES, J. \& MOLINA, O. (2011). ¿Por qué usamos las tecnologías de la información y la comunicación?. Revista Internacional de Sociología, 69 (2), 371-392. (doi:10.3989/ris.2010.01.15). 


\section{ANEXO 1}
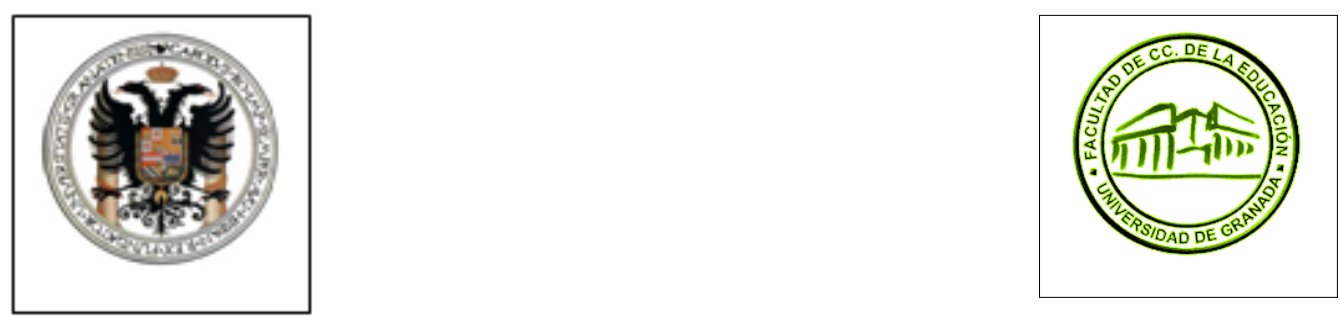

\section{EL PROFESOR DE MÚSICA DE ENSEÑANZA SECUNDARIA ANTE EL RETO ANDALUZ DE LA ESCUELA TIC 2.0.}

Con el fin de completar nuestra investigación, por favor, indique las opciones que usted considere oportunas en base a su experiencia.

\section{DATOS IDENTIFICATIVOS DEL PROFESORADO}

Sexo

(Seleccione una de las siguientes opciones)

Hombre

Mujer

\section{Edad}

(Seleccione una de las siguientes opciones)

menos de 25

25 a 34

35 a 44

45 a 55

más de 55

Años de experiencia docente

(Seleccione una de las siguientes opciones)

Menos de 1 año

1 a 5 años

6 a 10 años

11 a 15 años

16 a 20 años

Más de 20 años

Tipo de centro

(Seleccione una de las siguientes opciones)

Público

Concertado 
Provincia en la que imparte docencia

(Seleccione una de las siguientes opciones)

Almería

Cádiz

Córdoba

Granada

Huelva

Jaén

Málaga

Sevilla

Categoría profesional

(Seleccione una de las siguientes opciones)

- Funcionario de carrera con destino definitivo en su centro

- Funcionario de carrera con destino definitivo pero en comisión de servicios

- Funcionario sin destino definitivo

- Interino

\section{FORMACIÓN ACADÉMICA}

Formación académica inicial

(Marque las entradas que correspondan)

Licenciatura en Historia y Ciencias de la Música (Musicología)

Profesor Superior de Música

Grado Medio de Música o Enseñanza Profesional de Música

Grado Elemental de música o Enseñanza Elemental de Música

Magisterio especialidad Educación Musical

Otras (indique cuáles):

¿Cuál de estas asignaturas ha cursado durante su formación inicial? (indique una o varias opciones)

(Marque las entradas que correspondan)

Música y nuevas tecnologías

Composición con medios electroacústicos e informáticos

Tecnología informática aplicada a la música

Flamenco y nuevas tecnologías

Otras (indique cuáles):

Ninguna de ellas

\section{FORMACIÓN EN NUEVAS TECNOLOGÍAS}

[A partir de aquí marque una $\mathrm{X}$ teniendo en cuenta que 1.Nada, 2.Poco, 3.Suficiente, 4.Bastante, 5. Mucho].

Indique el grado de conocimiento y/o habilidad que posee usted sobre el manejo de las siguientes aplicaciones:

\begin{tabular}{|l|l|l|l|l|l|}
\hline \multicolumn{1}{c|}{} & 1 & 2 & 3 & 4 & 5 \\
\hline Manejo del correo electrónico & & & & & \\
\hline Marcadores sociales online (Delicious, misterwrong, meneame) & & & & & \\
\hline Blogs & & & & & \\
\hline
\end{tabular}




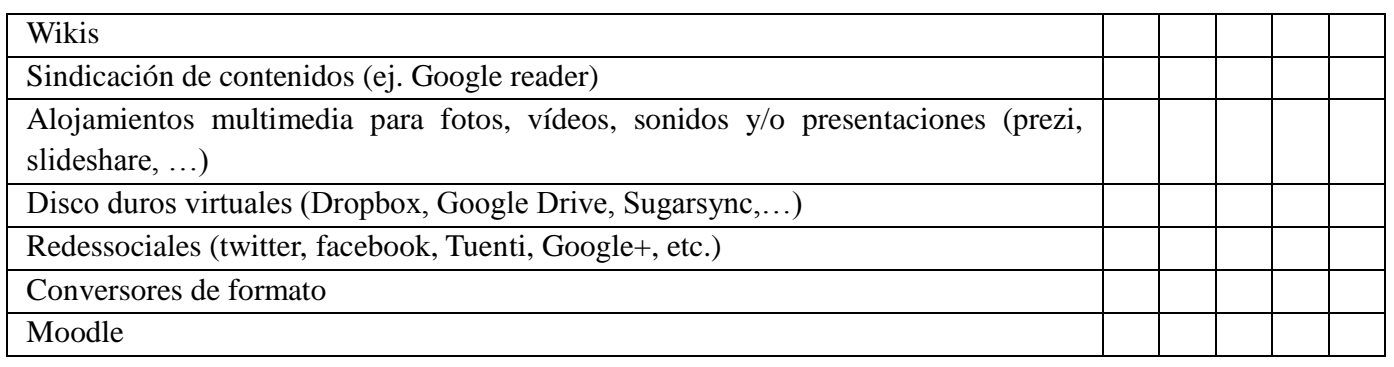

Determine en qué medida conoce usted los siguientes programas:

\begin{tabular}{|l|l|l|l|l|l|}
\hline \multicolumn{1}{l|}{} & 1 & 2 & 3 & 4 & 5 \\
\hline Editores de partituras & & & & & \\
\hline Secuenciadores & & & & & \\
\hline Editores de vídeo & & & & & \\
\hline Editores de imágenes & & & & & \\
\hline Generadores de arreglos MIDI (tipo band in a box) & & & & & \\
\hline
\end{tabular}

Señale el grado de conocimiento que tiene usted sobre los siguientes programas:

\begin{tabular}{|l|l|l|l|l|l|}
\hline Sibelius & 1 & 2 & 3 & 4 & 5 \\
\hline Musescore & & & & & \\
\hline Lilypond & & & & & \\
\hline Finale & & & & & \\
\hline Audacity & & & & & \\
\hline Rosegarden & & & & & \\
\hline Cubase & & & & & \\
\hline Avidemux & & & & & \\
\hline Cinelerra & & & & & \\
\hline Kolorpaint & & & & & \\
\hline GNUpaint & & & & & \\
\hline Gimp & & & & & \\
\hline
\end{tabular}

¿Cómo valora sus conocimientos y aptitudes sobre las TIC?

Sus conocimiento y aptitudes tic

\section{TECNOLOGÍAS EN EL AULA}

Señale en qué medida considera usted necesario contar con el siguiente equipamiento tecnológico en el aula de música:

\begin{tabular}{|l|l|l|l|l|l|}
\hline \multicolumn{1}{l|}{} & 1 & 2 & 3 & 4 & 5 \\
\hline Pizarras digitales interactivas & & & & & \\
\hline Ordenador para el profesor & & & & & \\
\hline Proyector & & & & & \\
\hline Internet & & & & & \\
\hline
\end{tabular}


Ordenadores disponibles para el uso del alumnado

Tableta, ebook, etc.

Equipo de sonido

Equipo de grabación digital de audio

Mesa de mezclas

Micrófonos

Televisor

Reproductor de DVD/Blue Ray/ y otros formatos multimedia

Indique en qué medida considera usted necesario contar con los siguientes recursos en el aula de música

\begin{tabular}{|c|c|c|c|c|c|}
\hline & 1 & 2 & 3 & 4 & 5 \\
\hline \multicolumn{6}{|l|}{ Instrumental Orff } \\
\hline \multicolumn{6}{|l|}{ Flautas } \\
\hline \multicolumn{6}{|l|}{ Piano } \\
\hline \multicolumn{6}{|l|}{ Guitarra } \\
\hline \multicolumn{6}{|l|}{ Caja Flamenca } \\
\hline Otros instrumentos musicales & & & & & \\
\hline
\end{tabular}

En su actividad docente, señale el grado de utilización que hace de las siguientes infraestructuras tecnológicas:

\begin{tabular}{|c|c|c|c|c|c|}
\hline & 1 & 2 & 3 & 4 & 5 \\
\hline Pizarras digitales & & & & & \\
\hline Internet & & & & & \\
\hline Proyector & & & & & \\
\hline Ultraportátiles o netbooks & & & & & \\
\hline Reproductor multimedia (mp3, DVD, etc) & & & & & \\
\hline Equipos de tratamiento de sonido & & & & & \\
\hline
\end{tabular}

En referencia a las estrategias educativas integradoras de las TIC en su práctica docente, valore el grado de dedicación a las mismas:

Clase magistral y expositiva con apoyo de las tic (PDI o proyección)

Clase con libro digital

Trabajo colaborativo con apoyo del ordenador, usando software específico

Trabajo autónomo, cada uno con su pc, usando software específico

Exposiciones de estudiantes con apoyo de las tic

Clases a través de espacios virtuales como Moodle,Sakai, Blogs, etc.

Trabajo autónomo en casa usando las tic

En referencia a su actividad docente, indique el grado en el que usted utiliza las TIC para:

\begin{tabular}{|c|c|c|c|c|c|}
\hline & \\
\hline & 1 & 2 & 3 & 4 & 5 \\
\hline \multicolumn{6}{|l|}{ Escuchar música } \\
\hline \multicolumn{6}{|l|}{ Mostrar y seguir musicogramas } \\
\hline \multicolumn{6}{|l|}{ Acompañar canciones instrumentales/vocales } \\
\hline Cantar en karaoke & & & & & \\
\hline
\end{tabular}




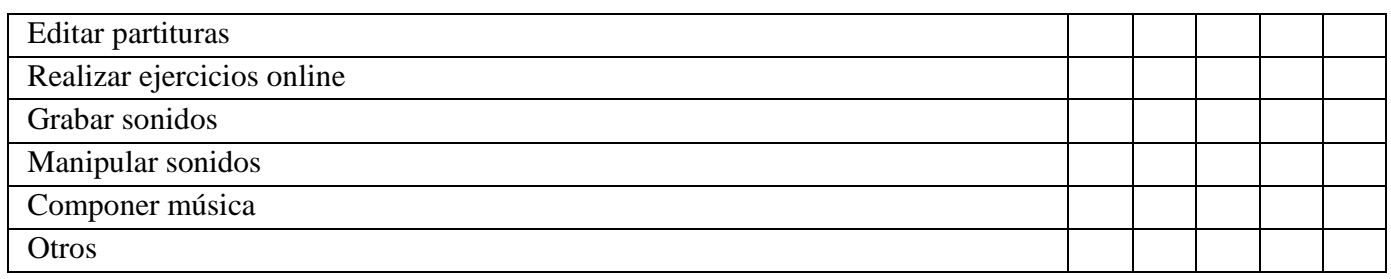

\section{ACTITUD HACIA LAS TIC Y COMPETENCIAS}

Valore el efecto de las TIC sobre el aprendizaje del alumnado:

\begin{tabular}{|c|c|c|c|c|c|}
\hline \multirow{2}{*}{ Están más motivados e implicados en las tareas de clas } & 1 & 2 & 3 & 4 & 5 \\
\hline & & & & & \\
\hline Han mejorado su rendimiento & & & & & \\
\hline Han desarrollado la competencia digital & & & & & \\
\hline Han mejorado la interpretación instrumental & & & & & \\
\hline Son más creativos & & & & & \\
\hline Trabajan más en equipo & & & & & \\
\hline Saben buscar mejor la información & & & & & \\
\hline Saben expresarse y comunicarse mejor & & & & & \\
\hline Han adquirido mejor los conocimientos de la materia & & & & & \\
\hline
\end{tabular}

Valore los obstáculos que conllevan las TIC en el aula:

\begin{tabular}{|c|c|c|c|c|c|}
\hline & 1 & 2 & 3 & 4 & 5 \\
\hline $\begin{array}{l}\text { Los problemas técnicos que a veces provocan me impiden el desarrollo normal } \\
\text { de las clases }\end{array}$ & & & & & \\
\hline Faltan medios que posibiliten el trabajo con las mismas & & & & & \\
\hline Requieren mucha inversión de tiempo para preparar cualquier material & & & & & \\
\hline Me falta preparación para poder utilizarlas convenientemente & & & & & \\
\hline $\begin{array}{l}\text { Aunque tengo la preparación suficiente para su uso, prefiero utilizar } \\
\text { metodologías tradicionales }\end{array}$ & & & & & \\
\hline
\end{tabular}

Valore sus actuaciones propias ante las TIC:

\begin{tabular}{|c|c|c|c|c|c|}
\hline & 1 & 2 & 3 & 4 & 5 \\
\hline Comparto con mis compañeros las experiencias donde utilizo las tic & & & & & \\
\hline $\begin{array}{l}\text { Investigo y reflexiono de las prácticas de aula, con el objeto de mejorar las } \\
\text { actuaciones tic frente al aprendizaje }\end{array}$ & & & & & \\
\hline $\begin{array}{l}\text { Planifico con tiempo las actividades con apoyo TIC, en relación con los } \\
\text { objetivos, el desarrollo de la tarea, selección y búsqueda de recursos }\end{array}$ & & & & & \\
\hline $\begin{array}{l}\text { Integro las TIC en la docencia, porque incrementa la calidad de los aprendizajes } \\
\text { de los estudiantes }\end{array}$ & & & & & \\
\hline
\end{tabular}

Valore usted en qué medida la utilización de las TIC ayudan al alumnado a:

\begin{tabular}{|l|l|l|l|l|l|}
\cline { 2 - 5 } \multicolumn{1}{c|}{} & 1 & 2 & 3 & 4 & 5 \\
\hline $\begin{array}{l}\text { Fomentar la capacidad de apreciar, comprender y valorar críticamente diferentes } \\
\text { manifestaciones }\end{array}$ & & & & \\
\hline Adquirir habilidades para expresar ideas, experiencias, etc. & & & & & \\
\hline
\end{tabular}




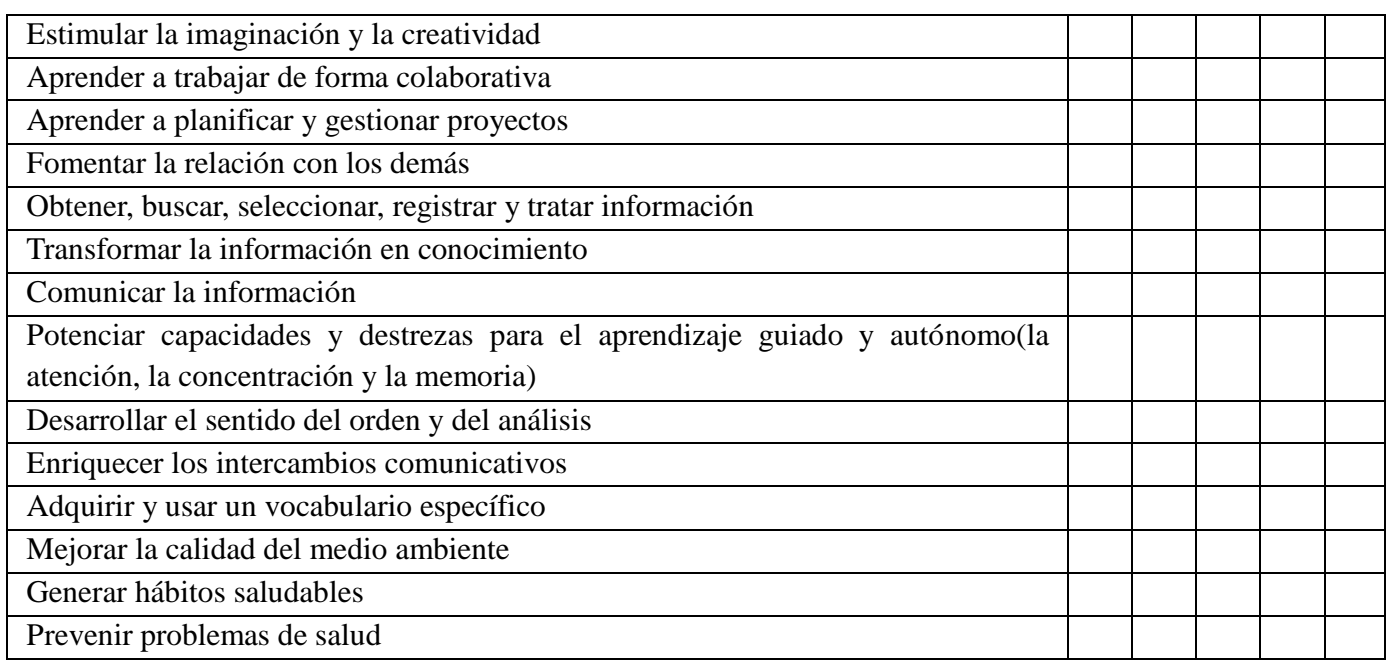

\section{RECURSOS TIC}

¿En qué medida le son útiles los recursos digitales de la mochila digital 2.0?

\begin{tabular}{|l|l|l|l|l|l|}
\cline { 2 - 6 } & 1 & 2 & 3 & 4 & 5 \\
\hline
\end{tabular}

¿En qué medida le son útiles los recursos digitales de la plataforma agrega?

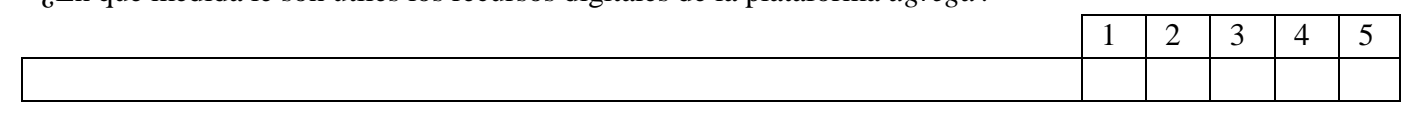

Valore el uso que hace de las siguientes aplicaciones en su aula:

\begin{tabular}{|l|l|l|l|l|l|}
\hline Moodle del aula & 1 & 2 & 3 & 4 & 5 \\
\hline Blog propio & & & & & \\
\hline Otros blogs & & & & & \\
\hline Página web propia & & & & & \\
\hline Otras webs & & & & & \\
\hline Plataforma agrega & & & & & \\
\hline Mochila digital 2.0 & & & & & \\
\hline Libro digital propio & & & & \\
\hline Libros digitales de editoriales & & & & & \\
\hline
\end{tabular}

Indique en qué medida considera usted necesario tener al alcance los siguientes recursos:

\begin{tabular}{|c|c|c|c|c|c|}
\hline & 1 & 2 & 3 & 4 & 5 \\
\hline $\begin{array}{l}\text { Plataforma específica del área de música con recursos tic ordenados por } \\
\text { contenidos curriculares }\end{array}$ & & & & & \\
\hline $\begin{array}{l}\text { Espacio web dedicado a la formación online en lo que a aplicaciones musicales } \\
\text { se refiere }\end{array}$ & & & & & \\
\hline Asistencia de un técnico en su centro & & & & & \\
\hline Un servicio de asistencia tic virtual que resuelva sus dudas al instante & & & & & \\
\hline
\end{tabular}


VII. LA ESCUELA TIC 2.0 Y LAS FAMILIAS

Señale en qué grado considera usted que:

\begin{tabular}{|c|c|c|c|c|c|}
\hline \multirow[b]{2}{*}{ Los ordenadores portátiles son adecuadamente cuidados por el alumnado } & 1 & 2 & 3 & 4 & 5 \\
\hline & & & & & \\
\hline \multicolumn{6}{|l|}{$\begin{array}{l}\text { Los/as alumnos/as reciben colaboración por parte de sus familias en las tareas } \\
\text { digitales propuestas por usted }\end{array}$} \\
\hline \multicolumn{6}{|l|}{$\begin{array}{l}\text { Los/as alumnos/as reciben la supervisión de sus padres a la hora de utilizar } \\
\text { Internet }\end{array}$} \\
\hline $\begin{array}{l}\text { Se ha ofertado formación suficiente para las familias en relación al uso adecuado } \\
\text { de las tic, bien desde el centro, bien desde otras instituciones }\end{array}$ & & & & & \\
\hline
\end{tabular}

Cualquier otro comentario que usted considere oportuno

Gracias por su colaboración

\section{Correspondencia con los autores}

Emilia MARTOS SÁNCHEZ

$\mathrm{C} /$ Antonio Machado, $\mathrm{n}^{\circ} 8$

04867 Macael (Almería)

e-mail: martosemi@gmail.com

Purificación PÉREZ-GARCÍA

Facultad de Ciencias de la Educación

Campus Universitario de Cartuja s/n

18071 Granada (Spain)

e-mail:mpperez@ugr.es

Julia BERNAL VÁZQUEZ

C/ Pedro Antonio de Alarcón, n ${ }^{\circ} 37,5^{\circ} \mathrm{A}$

18004 Granada

e-mail: jbernal@ugr.es 